\title{
exemple de rupture de fondations ancrées par tirants précontraints
}

\section{an exemple of rupture of prestressed ground anchored foundations}

\author{
T.R.A. SANGLERAT \\ Ingénieur I.N.S.A., Master of Science* \\ G. SANGLERAT** \\ Professeur de Mécanique des Sols à l'Ecole Centrale de Lyon \\ Expert près la Cour d'Appel de Lyon \\ Agréé par la Cour de Cassation
}

\section{Résumé}

La stabilité des fondations d'un très grand hall d'exposition était assurée par des tirants précontraints ancrés dans le sol. La rupture de trois d'entre eux a failli provoquer la ruine de l'ouvrage. On décrit la structure de l'édifice, les modalités de la rupture des tirants due à une protection insuffisante contre la corrosion, ainsi que les travaux confortatifs réalisés qui ont atteint 7.616.412 francs français.

\section{Abstract}

A case study involving failure of prestressed ground anchors used to support an Exhibition Hall is described. The design of the structure featured a 200-foot span cable supported fabric roof. Details of the structure and design of the foundation system are presented. Failure of the ground anchors caused the cable-supported roof to partially collapse. Investigation of the failure mechanism showed that inadequate anchor grouting and subsequent corrosion of steel in the anchors caused failure. Remedial measures costing over three-fourths million dollars are described. 


\section{INTRODUCTION}

Les travaux rapportés ici sont relatifs à un Palais d'Exposition construit en 1968 , de $100 \mathrm{~m}$ de long et de $82 \mathrm{~m}$ de large avec une travée libre de $60 \mathrm{~m}$. Sa couverture repose sur deux réseaux de câbles souples prenant appui sur des portiques en charpente métallique ancrés chacuns par deux tirants précontraints encastrés dans le sol.

En janvier 1980, au cours de ses opérations de nettoyage, un balayeur a trouvé deux boulons sur le plancher du hall d'exposition. Plutôt que de jeter ces deux pièces métalliques avec les autres détritus, cet ouvrier a alerté son chef d'équipe en se disant que probablement, ces boulons provenaient de la charpente métallique, ce qui constituait un fait anormal. Ce chef d'équipe a signalé l'incident au service de sécurité de la ville.

Grâce à cette présence d'esprit, un accident grave a pu être évité. En effet, on a alors constaté que la charpente métallique s'était fortement déformée au droit de trois de ses appuis qui étaient pratiquement à la limite de la rupture. Une surcharge de neige, même faible, aurait pu provoquer l'effondrement de la construction.

L'objet de la présente publication est de décrire l'ouvrage, de rappeler l'historique des travaux, d'analyser les causes des désordres et de commenter les travaux confortatifs qui ont fait l'objet d'une expertise judiciaire confiée à l'un des auteurs par le Tribunal de Grande Instance de la ville.

\section{DESCRIPTION DE L'OUVRAGE}

Il s'agit d'un ouvrage important de $100 \mathrm{~m}$ de long sur $82 \mathrm{~m}$ de large d'une conception relativement audacieuse, puisque sur une portée de $60 \mathrm{~m}$, sa partie centrale est dégagée de tout point porteur (fig. 1).

La couverture (étanchéité sur panneaux isolants et bacs d'aciers galvanisés) prend appui, ainsi que l'installation électrique, sur un double réseau de câbles comportant un nappe supérieure "porteuse" et une nappe infërieure dite "anti-soulèvement". Ces deux nappes reliées par une triangulation constituée de ronds à béton reposent sur vingt portiques en charpente métallique. Cette surface à câbles a été réalisée selon le procédé Jawerth.

Les tractions exercées par ces câbles sont reprises par des chevalements métalliques de forme triangulaire de $14,30 \mathrm{~m}$ de haut et de $10,72 \mathrm{~m}$ de base dont la coupe est donnée par la figure 2.

La charge verticale transmise par ces chevalements métalliques est reprise par des pieux qui traversent des remblais miniers pour s'encastrer vers $12 \mathrm{~m}$ de profondeur dans du schiste houiller. Ces pieux forés en béton armé $(\varnothing 130 \mathrm{~cm}$ côté intérieur et $\varnothing 110 \mathrm{~cm}$ côté extérieur) sont reliés en tête par une poutre traverse en béton armé.
Pour éviter le soulèvement des pieux extérieurs (fig. 3), il a été prévu des ancrages précontraints. La base extérieure du chevalement de chaque portique est ainsi retenue par deux câbles précontraints traversant le massif d'assise dans des gaines tube acier.

Les travaux de fondations comprenaient:

- cinquante-huit pieux de diamètres allant de $0,60 \mathrm{~m}$ à $1,30 \mathrm{~m}$;

- quarante tirants précontraints à sceller au rocher dont trente-six équipés de quarante-quatre fils ou brins plats de type $\mathrm{KA}$ et quatre équipés de vingt-huit fils $\mathrm{KA}$ (pour les portiques d'angles).

Ces fils KA, fabriqués en Allemagne sont constitués d'étroits fers plats crantés simplement juxtaposés. Ils ne se présentent donc pas sous forme de torons.

Pour permettre aux magistrats de déterminer les responsabilités encourues et de ventiler le coût de la réparation des désordres entre les différents intervenants, la première tâche fut de rechercher quel avait été exactement le déroulement des travaux.

\section{DÉROULEMENT DES TRAVAUX}

La conception architecturale initiale, qui prévoyait une ossature du Palais des Expositions constituée par des portiques métalliques de $60 \mathrm{~m}$ de portée, articulés en pied et encastrés en tête, a été modifiée sur proposition de l'entreprise qui a préconisé l'exécution d'une structure à câbles suivant le procédé Jawerth.

L'entreprise a défini la conception des fondations d'une manière très détaillée: nombre de pieux, diamètres et longueurs, ainsi que le type des tirants d'ancrage (procédé KA), leur nombre et leur section.

Les pieux forés ont été exécutés en mai et juin 1968. Le forage, la mise en place et le scellement au rocher (schiste minier) des tirants ont été réalisés en juin et juillet 1968 , la protection contre la corrosion étant effectuée ultérieurement à l'aide d'un coulis de ciment.

Lors des essais de mise en tension, exécutés en août 1968, il est apparu qu'une partie des tirants était incapable de reprendre les charges pour lesquelles ils avaient été prévus, en raison de la mauvaise qualité du terrain d'ancrage minier. Une galerie de mine «inconnue" (ou plutôt "oubliée») a êté découverte à ce moment-là.

Pour permettre l'ouverture de la $1^{\text {re }}$ foire en septembre 1968, ce qui était un impératif absolu du maître d'ouvrage, l'entreprise a proposé les nesures temporaires suivantes:

- maintien des tirants scellés au rocher dans les zones centrales, avec les tensions partielles obtenues;

- réalisation de quatre massifs d'angle complémentaires en béton coulés dans le sol pour retenir le déficit de tension des tirants pour la durée de la foire seulement. Ces massifs d'angle ont été exécutês entre le 6 septembre et le 25 septembre 1968. 


\section{PALAIS DES EXPOSITIONS}

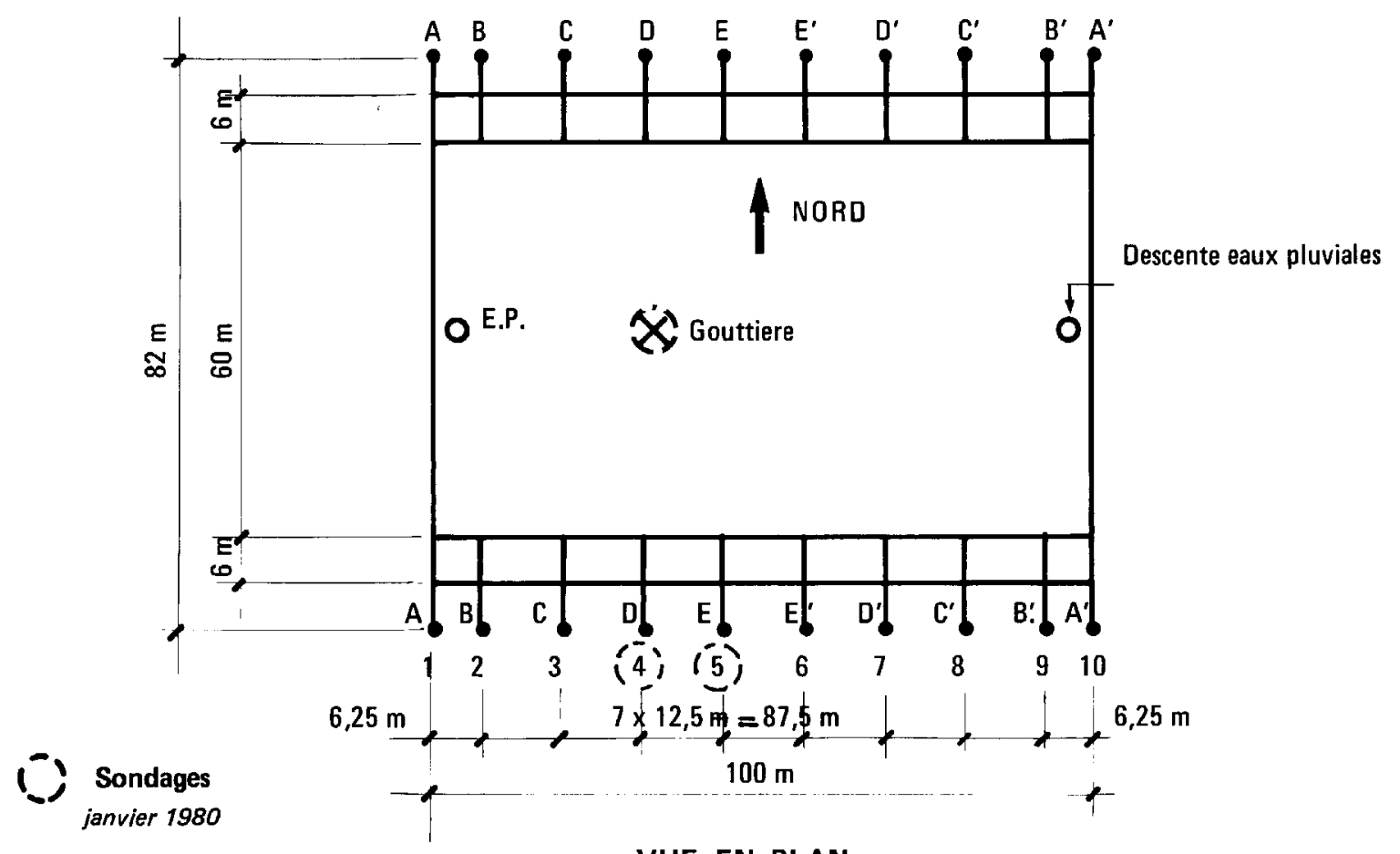

VUE EN PLAN

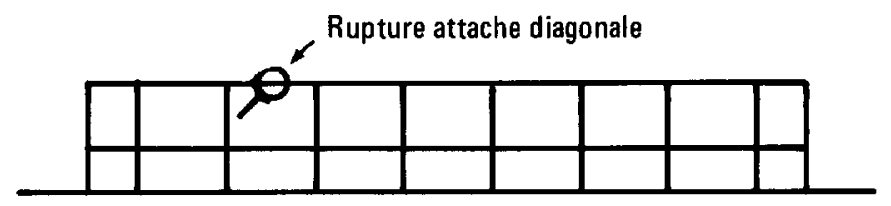

ELEVATION SUD

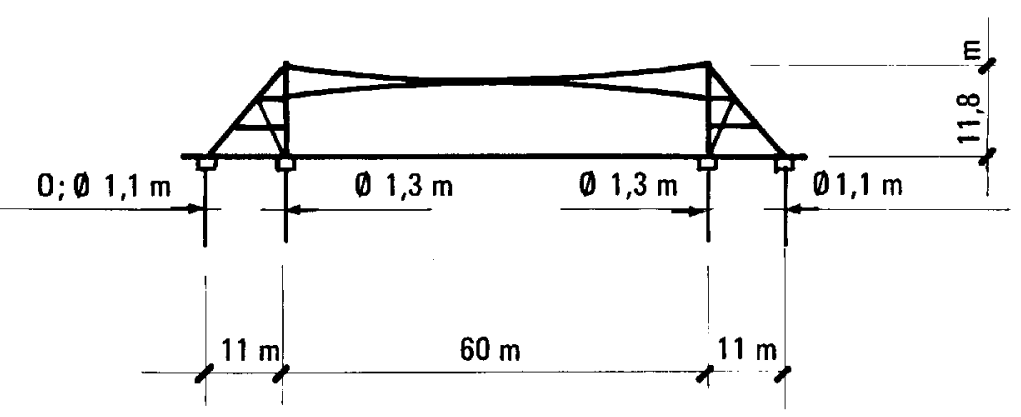

COUPE NORD - SUD

Fig. 1 


\section{SITUATION INITIALE}

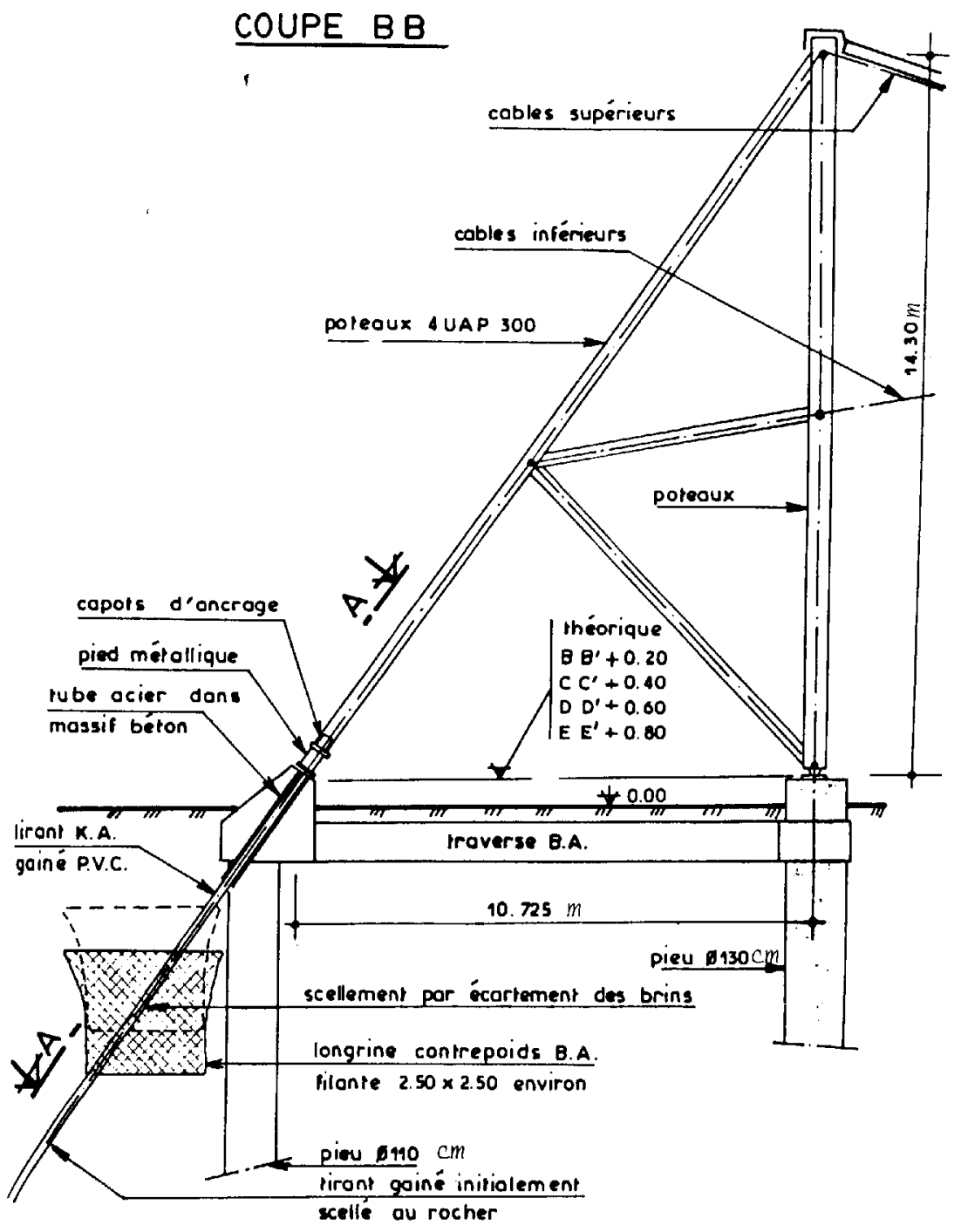

Fig. 2
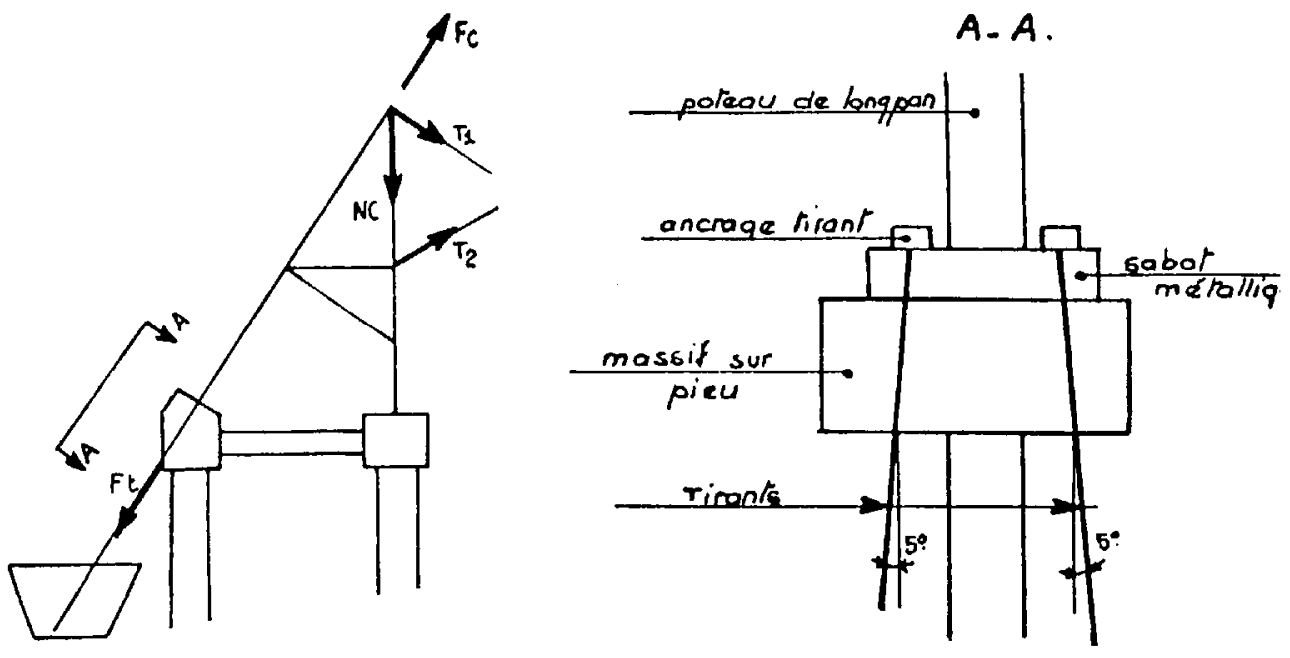

Fig. 3 
Après le déroulement de la foire, pour améliorer les ancrages à titre définitif, il a été décidé de réaliser une longrine en béton armé (schématisée sur les figures 2 et 4 par les zones grisées) destinée à jouer le rôle de contrepoids. On a donc enrobé les tirants, dans cette longrine poids, le scellement se faisant par écartement des brins.

\section{SITUATION INITIALE}

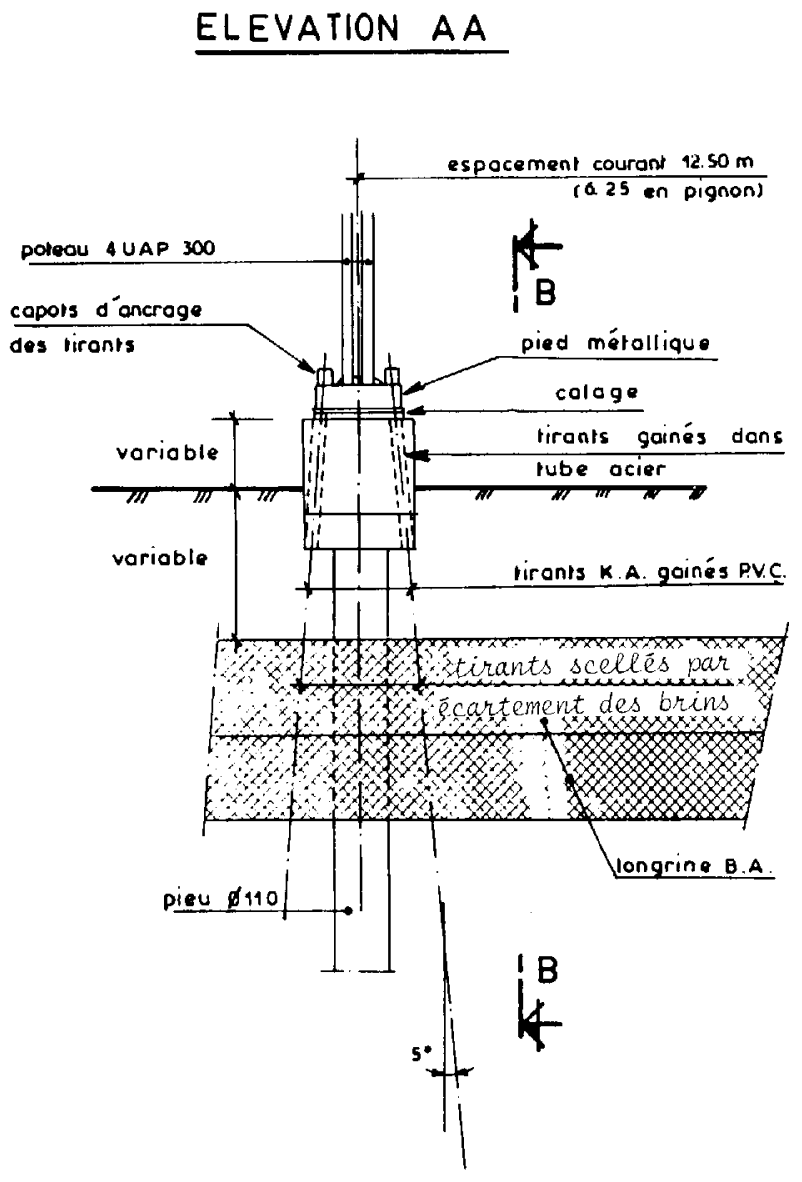

Fig. 4

Le schéma type de protection des tirants initiaux du type $\mathrm{KA}$ avec quarante-quatre brins est indiqué par la figure 5 .

En février 1969, on a réalisé une campagne d'injections destinée à remplir les vides sous les assises des pieux. Les travaux ont fait l'objet d'une réception provisoire le 17 mars 1969 et d'une réception définitive le 18 septembre 1969

La présence d'un tube métallique d'injection n'avait pas permis l'accrochage de trois tirants précontraints aux massifs $C$, D et ' $C^{\prime}$ en long pan Nord (fig. 1).

\section{SCHEMA TYPE DE PROTECTION \\ DES TIRANTS INITIAUX}

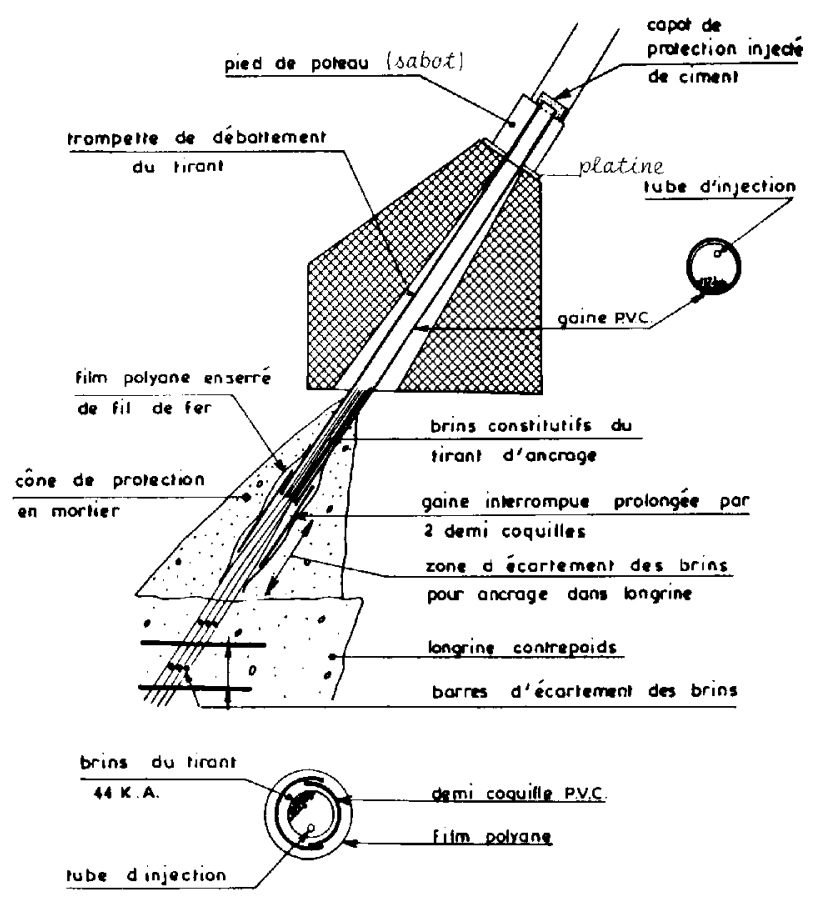

Fig. 5

Ces trois tirants, restés scellés au rocher depuis 1968 sans injection pendant deux ans, ne présentaient plus une sécurité normale vis-à-vis de la corrosion. En juin et juillet 1970, il a donc été demandé à l'entreprise de mettre en place en remplacement trois nouveaux tirants.

Enfin le bureau de contrôle, qui n'avait qu'une mission très limitée, a demandé également de mettre en place deux tirants supplémentaires sur le massif E' Sud afin de permettre le prélèvement d'échantillons du câble initial pour examen en laboratoire en vue d'évaluer ses caractéristiques. Ces examens n'ont mis en évidence aucune anomalie.

Ces nouveaux tirants, mis en place deux ans après les tirants KA, sont de type B.B.R.V.

\section{DÉSORDRES OBSERVÉS}

Dès que nous avons été saisi, en janvier 1980, d'une mission d'expertise par le Tribunal de Grande Instance, nous avons examiné les lieux litigieux contradictoirement en présence des parties: maître d'ouvrage, entreprises et architecte assistés par leurs avocats. 


\subsection{Désordres observés au-dessus de la surface du sol}

Des désordres particulièrement importants affectent les pièces d'appui métalliques des poteaux' de chevalement situés au-dessus des longrines en tête des pieux. Les déformations les plus importantes étaient notées en façade Sud pour les portiques D, E et D', dont les soulèvements variaient de 6 à $13 \mathrm{~cm}$, d'où des déchirures des âmes et membrures malgré leur très forte épaisseur. Fort heureusement, les galeries de circulation en mezzanine à l'intérieur du hall en façades Sud et Nord ont joué le rôle de poutres de répartition des efforts, ce qui a limité l'amplitude des déformations en reportant les efforts des travées les plus sollicitées par les ruptures sur les travées adjacentes. Par suite du mouvement des fondations, les câbles supportant la toiture s'étaient déformés, d'où des déchirures dans l'étanchéité et création de poches de rétention d'eau surchargeant dangereusement la toiture.

Lors de l'examen détaillé des différentes pièces constituant la charpente métallique, nous avons constaté de très nombreux défauts d'exécution, l'entreprise n'ayant pas respecté les plans approuvés par le bureau de contrôle.

\subsection{Constatations au-dessous du niveau du sol}

Les déformations graves observées dans certains portiques provenaient à l'évidence, soit de déformations excessives des tirants précontraints, soit de la rupture de certains d'entre eux pour au moins trois des portiques de la façade Sud. Il était donc indispensable d'examiner l'état des tirants précontraints enterrés.

Nous avons donc fait procéder à deux sondages à ciel ouvert par puits blindés pour mettre à nu le massif d'ancrage ainsi que la longrine de béton formant contrepoids qui devait avoir $2,50 \mathrm{~m}$ de large et $2,50 \mathrm{~m}$ de hauteur.

Toute surcharge de neige aurait pu conduire à l'effondrement brutal de l'ensemble du bâtiment et comme on ne savait pas si d'autres tirants n'étaient pas sur le point de se rompre avant d'entreprendre les sondages, un élingage des chevalements sinistrés fut réalisé en ancrant les élingues dans des massifs de béton coulés à l'extérieur du bâtiment. En outre, les câbles supportant la couverture furent partiellement détendus pour améliorer la sécurité de l'ouvrage.

Avant de commencer ces investigations, nous pensions que les câbles précontraints s'étaient rompus dans le sol par corrosion. En effet, des analyses chimiques avaient démontré l'agressivité de l'eau prélevée dans ce sol minier.

Le sondage réalisé au droit du massif $D$ Sud a mis en évidence un soulèvement. Il a été constaté que des blocages béton avaient été réalisés autour des tirants, entre le massif d'appui des piliers métalliques et la longrine contrepoids (fig. 8).
Près de la surface de la longrine contrepoids, on a observé un glissement de la gaine du tirant côté Ouest au niveau de la nappe phréatique. La qualité du béton des longrines contrepoids était très irrégulière.

Au droit du massif $E$ Sud, on a noté également une rupture de la gaine du tirant entre le dessus de la longrine et le massif sur pieux. Dans le tirant précontraint Est, on a observé deux brins corrodés et cassés.

D'une manière gënêrale, les gaines P.C.V. étaient souvent déchirées ou même éclatées, l'enrobage des brins par le coulis ciment très irrégulier et pratiquement inexistant en certains endroits. Les corrosions de certaines cassures ont montré que celles-ci étaient anciennes.

On a constaté que de nombreux brins de tirants étaient rouillés, et quelquefois, sans aucune protection de coulis d'injection.

La figure 4 montre la position des tirants $\mathrm{KA}$ placés dans des gaines P.V.C. en partie inférieure et gainés dans des tubes acier en partie supérieure à travers le massif de fondations reposant sur la tête des pieux. La figure 5 précise le schéma type de protection des tirants initiaux.

Au-dessus des longrines contrepoids, la gaine enveloppant chaque tirant était interrompue et prolongée par deux demi-coquilles enveloppées par un pochon de béton non armé en íorme de cône (fig. 5 et 6). La figure 6 donne le schéma de principe de renforcement envisagé initialement après l'exécution des deux premiers sondages, et qui a dû être modifié ultérieurement.

Il paraît intéressant de donner quelques précisions sur les contraintes subies par les tirants $\mathrm{KA}$ d'origine.

Les tractions $T_{1}$ et $T_{2}$ des câbles de toiture engendrent une traction $F_{C}$ dans les poteaux du long pan du chevalement métallique et une compression $\mathrm{N}_{\mathrm{C}}$ du poteau métallique vertical (fig. 3 ). La traction $F_{C}$ est reprise par les tirants d'ancrage tendus dans le sol avec une traction $F_{t}$. Suivant les cas de charge, $F_{C}$ varie de 2976 à $3871 \mathrm{kN}$. Les tirants sont composés de quarante-quatre fils $\mathrm{KA}$ (section totale $1707 \mathrm{~mm}^{2}$ ). Ils devaient pouvoir encaisser (fig. 3) chacun un effort de:

$\frac{3871}{2 \times \cos 5^{\circ}}=1943 \mathrm{kN}$ correspondant à une
tension de: $\frac{1943}{1707}=1,138 \mathrm{kN} / \mathrm{mm}^{2}=1138 \mathrm{MPa}$

Cette tension correspond à $81 \%$ de la limite élastique de $1410 \mathrm{MPa}$ garantie par les fils KA.

Cela dépasse les $60 \%$ imposés par les règles de lart, puis par une recommandation Securitas de 1972 (règles dites TA 72).

Normalement, les constructeurs auraient dû tendre les tirants d'ancrage avant les câbles de toiture, c'est-à-dire appliquer en tête du portique pieux-traverse un effort 


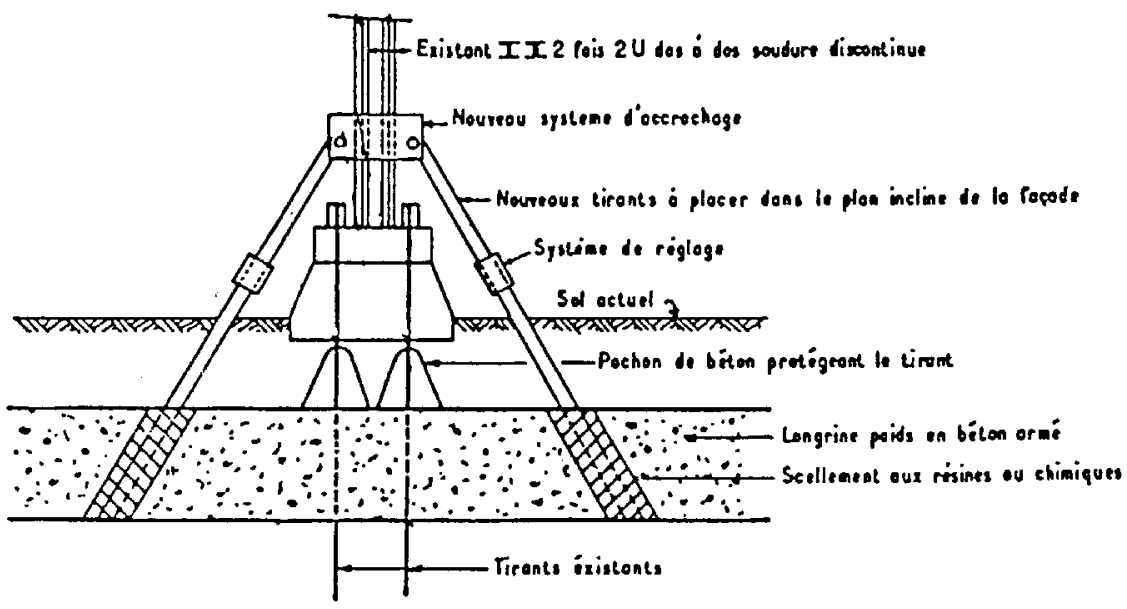

SCHEMA DE PRINCIPE D'UN RENFORCEMENT

Fig. 6

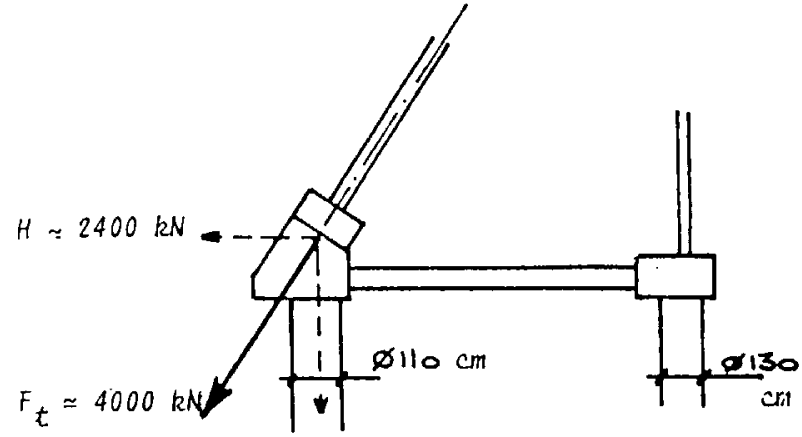

Fig. 7

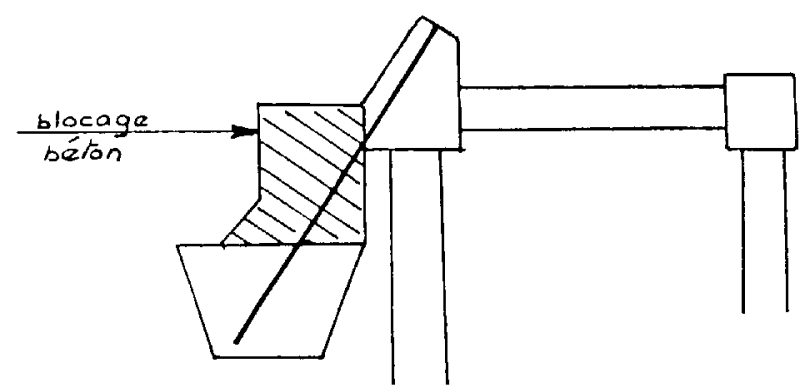

Fig. 8
$F_{\text {t de }} 4000 \mathrm{kN}$ environ, d'où une composante horizontale $\mathrm{H}=2400 \mathrm{kN}$. Or, ce portique ne peut supporter en service normal qu'une charge horizontale $\mathrm{H}$ de l'ordre de $700 \mathrm{kN}$.

On voit que la mise en tension préalable des tirants ne pouvait s'effectuer sans entraîner la rupture des pieux. Il est donc vraisemblable, et ceci a été confirmé, que l'entreprise a tendu des tirants, puis des câbles de charpente, par paliers successifs, afin de limiter les sollicitations des pieux. Notons cependant que des traces de déplacement des têtes de pieux étaient visibles dans le revêtement de sol au moment de l'expertise.

Une telle opération ne pouvant se faire sur l'ensemble des tirants, il y a eu forcément des reports de charges transmis par la charpente de chevalement à chevalement. Ceci explique vraisemblablement les traces de plastification anciennes observées sur les goussets horizontaux les plus faibles de la charpente.

Une fois la structure terminée, les tirants ont été scellés à la base des poteaux et leur tension ne pouvait plus être modifiée. Cela aurait donc posé des problèmes graves si l'on avait voulu détendre les câbles de la toiture, ne serait-ce que pour les changer; à ce moment-là, la tête des pieux n'aurait plus été équilibrée et les pieux auraient pu se rompre. Du reste, nous avons observé qu'au droit des tirants remplacés en 1970 (C Nord, D Nord, C' Nord, E' Sud), l'entreprise a disposé de volumineux blocages en béton entre les massifs sur pieux et la longrine contreproids, comme indiqué par la figure 8 . Ces blocages appuient les têtes de pieux sur la longrine et limitent leur déplacement. On peut donc tendre les tirants indépendamment des forces induites par la toiture au droit des chevalements correspondants.

\section{ORIGINE ET CAUSES DES DÉSORDRES}

\subsection{Origine des désordres}

Une fois les travaux confortatifs réalisés (cf. § 6), la structure présentant un coefficient de sécurité normal, il a été possible de procéder à l'inspection détaillée des tirants litigieux, tant en long pan Sud qu'en long pan Nord, afin de localiser les zones de rupture d'ancrages précontraints, qui étaient à l'origine du sinistre. 
La rupture de tirants précontraints peut avoir des origines diverses:

- Rupture des scellements, soit par insuffisance de l'injection de scellement, soit en raispn d'une longueur insuffisante de la zone scellée en égard à la qualité réelle du terrain encaissant.

- Rupture de la tête du tirant.

- Rupture des fils constituant le tirant.

C'est manifestement à ce dernier type de rupture qu'est imputable le sinistre. En effet, les sondages visuels effectués ont mis en évidence les faits suivants:

a. De nombreux défauts d'injection en plusieurs parties des tirants et notamment près de leur extrémité supérieure.

b. Le tirant Ouest du massif $\mathrm{D}$, le premier tirant qui a lâché (et dont la rupture a déclenché l'apparition de la plupart des désordres observés) montre que, contrairement à ce qu'on avait pensé à l'origine, la zone la plus fortement corrodée des fils ne se trouve pas en contact avec le sol, ni même avec la nappe phréatique, mais est située à la partie supérieure des tirants, près du sabot métallique et de son support en béton.

La zone de rupture du tirant se situe sensiblement entre 20 et $80 \mathrm{~cm}$ sous la base de la platine des plaques d'appui du sabot de la charpente métallique, c'est-à-dire au-dessus du sol, donc à l'air libre.

Dans cette zone, aucun coulis ne protégeait les fils.

c. Les constatations faites pour le tirant précédent se retrouvent pratiquement systématiquement pour tous les autres tirants.

d. La jonction de deux gaines plastiques bout à bout avec l'aide de deux demi-coquilles ne permettait pas d'assurer une injection correcte (fig. 5).

Enfin, toutes les parties ont été unanimes pour reconnaître que toutes les injections d'origine des trants KA ont été faites sans pression c'est-à-dire uniquement par gravité. Précisons que cette mise en cuvre acceptée à l'époque, est abandonnée aujourd'hui, car l'expérience a montré qu'elle était incapable d'assurer une pénétration suffisante, donc une protection correcte et efficace à long terme contre la corrosion.

\subsection{Causes des désordres}

Il n'a jamais été observé de rupture des fils KA par striction. Il ne s'agit donc pas d'un dépassement de la limite de rupture imputable, par exemple, à une erreur de dimensionnement des tirants. Par contre, les nombreux examens visuels confirmés par les analyses métallographiques effectuées à l'Ecole Centrale de Lyon, montrent à l'évidence que les très nombreuses ruptures des fils de précontrainte du type KA observées sont dues au phénomène de corrosion fissurante sous tension. Il s'agit d'une rupture qui s'effectue sans modification de la section du fil. La corrosion fissurante sous tension résulte d'un phénomène électrolytique. Un effet de pile se produit à la suite de la création d'une différence de potentiel entre deux points du fil considéré, provenant d'une aération différentielle du métal.
La corrosion anodique correspondant à une fusion du métal est extrêmement rare. La corrosion cathodique est la plus courante, elle se produit en présence d'un électrolyte qui est, généralement, l'eau. Il y a alors dégagement d'hydrogène naissant qui pénètre dans l'acier en augmentant le volume des cristaux constituant l'acier, ce qui provoque des micro-fissures. C'est pourquoi cette corrosion est dite "fissurante"

A partir d'un point de la surface, les micro-fissures se développent et pénètrent de plus en plus à l'intérieur de la section de l'acier en provoquant un véritable effet d'entaille. En conséquence, la section résistante devenant de plus en plus faible, la limite de rupture décroît avec le temps et peut être atteinte sous la contrainte de service.

Ce phénomène de corrosion fissurante sous tension est d'autant plus accentué que par suite de la traction initiale des aciers, très élevée dans le cas des câbles précontraints, les cristaux d'acier sont relativement distendus ce qui facilite la progression de la corrosion interne.

Cette corrosion sous tension est imputable elle-même à deux causes concomitantes:

- L'une, principale: absence de protection par coulis d'injection sur certains tronçons des tirants.

- L'autre, secondaire: la nature même des aciers.

Il s'agit, en effet, d'aciers KA d'origine allemande, dont l'expérience a montré qu'ils étaient extrêmement sensibles au phénomène de corrosion sous tension. De nombreuses ruptures de tirants précontraints composés de fils KA ont été observées dans plusieurs pays. Depuis lors, cette nuance d'aciers est refusée pour tout tirant précontraint, alors qu'en 1968, elle était admise.

Les ruptures des fils ont toujours été observées dans les zones sans coulis. Dans ce cas, les zones non protégées étant en partie haute, au-dessus du sol, ni l'agressivité du terrain, ni l'agressivité des eaux de la nappe phréatique n'ont ici joué un rôle dans les ruptures observées.

Dans une certaine mesure, on peut classer le sinistre dans la catégorie que nous appelons «rançon du progrès technique». En effet, à une période donnée, on utilise des matériaux et des processus d'exécution qui n'ont pas encore fait leurs preuves à long terme et qui doivent être abandonnés quelques années plus tard en raison des désordres observés.

\section{TRAVAUX CONFORTATIFS}

En application des textes législatifs et réglementaires définis par le nouveau code de procédure civile français, l'expert judiciaire ne peut assurer aucune maitrise d'œuvre de travaux confortatifs.

En conséquence, nous avons demandé au maitre d'ouvrage de s'adjoindre un ingénieur conseil structures pour établir les plans détaillés des travaux confortatifs et assurer la direction de leur exécution. Ces travaux, 
qui ont été confiés à des entreprises autres que celles ayant participé à la construction initiale, ont comporté principalement :

1. L'abandon de tous les tirants d'ancrage existants supposés corrodés et risquant, à plus ou moins long terme, de se rompre. En effet, tous les tirants KA ayant été exécutés de la même manière, il n'y avait aucune raison pour que la corrosion fissurante sous tension ne fasse pas son oeuvre à plus ou moins longue échéance.

2. La mise en place par Soletanche Entreprise de nouveaux tirants précontraints, type barres Dywidag, ancrés dans le massif contrepoids enterré existant. Pour chaque appui, il a été prévu 4 barres Dywidag $\varnothing$ $36 \mathrm{~mm}$ tendues à $700 \mathrm{kN}$ scellées au coulis Pagel dans le béton du massif contrepoids existant.

3. La superposition de longrines, minces, inclinées, en béton armé précontraintes, au massif contrepoids de béton non armé. En effet, au cours des travaux confortatifs, on a découvert que le massif contrepoids en béton non armé existant avait une section plus faible que prévue sur les plans, ce qui entraînait des excentrements d'efforts. Comme le béton qui le constituait était souvent de très mauvaise qualité, il est donc apparu néccessaire de lester ce contrepoids et de modifier le système d'ancrage. Chacune de ces nouvelles longrines a été précontrainte par 10 barres Dywidag $\varnothing 26,5 \mathrm{~mm}$ gainées et injectées au brai epoxy et tendues à $400 \mathrm{kN}$.

4. La mise en place de bretelles de liaison entre les poteaux et les nouveaux tirants (fig. 9).

5. La vérification et réparation de la charpente dans la zone sinistrée.

Les figures 10 et 9 présentent respectivement l'élévation et la coupe au droit d'un chevalement après achèvement des travaux confortatifs.

Les poteaux U.A.P. 300 ont été renforcés et il a été mis en place des goussets boulonnés H.R. (Haute Résistance) avec articulations hautes et basses reliées à deux bretelles métalliques réglables pour chaque appui.

Nous remercions le professeur ODROBINSKI qui, pendant son séjour à Lyon a participé aux opérations de contrôle du renforcement des fondations et de la structure métallique.

Une première mise en sécurité provisoire de la structure avait été réalisée au début de l'expertise pour permettre l'utilisation du bâtiment pour la foire annuelle en septembre 1980. L'ensemble des travaux confortatifs définitifs a été achevé ensuite avant la foire de septembre 1981

Le coût des travaux confortatifs est ventilé poste par poste dans le tableau ci-après.

\section{SUITE JUDICIAIRE DE L'EXPERTISE}

Notre rapport d'expertise a été déposé au Tribunal de Grande Instance le 31 mars 1982.
Tableau du coût des travaux confortatifs

\begin{tabular}{|c|c|c|}
\hline Nature & \multicolumn{2}{|c|}{ Montants en francs T.T.C. } \\
\hline $\begin{array}{l}\text { Travaux prépara- } \\
\text { toires (sondages, lo- } \\
\text { cation échafaudage) }\end{array}$ & & 36234,77 \\
\hline $\begin{array}{l}\text { Travaux annexes } \\
\text { (chaussée, gaz, élec- } \\
\text { tricité) ..................... }\end{array}$ & & 233637,00 \\
\hline \multirow[t]{2}{*}{ 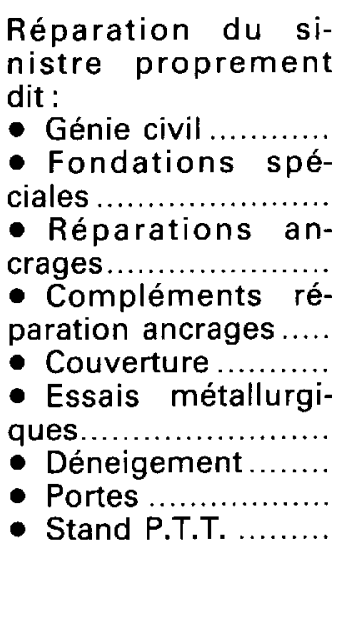 } & $\begin{array}{r}2472879,64 \\
723978,83 \\
1835394,96 \\
\\
360433,42 \\
318245,55 \\
\\
17372,93 \\
5123,71 \\
16274,70 \\
2646,00\end{array}$ & \multirow[b]{2}{*}{7752349,78} \\
\hline & & \\
\hline $\begin{array}{l}\text { Remise en état de la } \\
\text { boulonneriee............ }\end{array}$ & & 411600,00 \\
\hline \multirow[t]{2}{*}{ 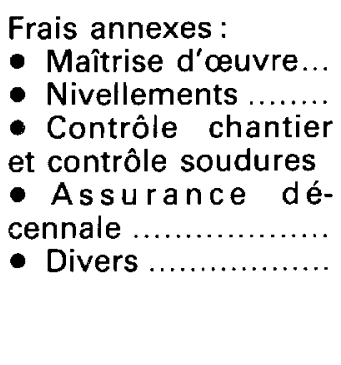 } & $\begin{array}{r}790792,97 \\
54501,87 \\
89376,00 \\
\\
135857,17 \\
113082,18\end{array}$ & \\
\hline & & 1183610,19 \\
\hline Total général. & & 7616412,23 \\
\hline
\end{tabular}

Nous avons appris récemment que le maître d'ceuvre demandeur n'a donné en l'état aucune suite judiciaire au fond de cette affaire. Peut-être parce que le délai de la garantie décennale était largement dépassé au moment de l'apparition des désordres en janvier 1980, puisque la réception définitive des travaux litigieux avait été prononcée le 18 septembre 1969.

\section{CONCLUSIONS}

1. Les désordres apparus en janvier 1980 auraient pu conduire à l'effondrement brutal de l'ossature à plus ou moins brève échéance.

2. Les désordres résultaient du soulèvement de trois fondations au droit des massifs $D, E$ et $D^{\prime}$ en façade Sud, d'où un basculement d'ensemble des chevale- 


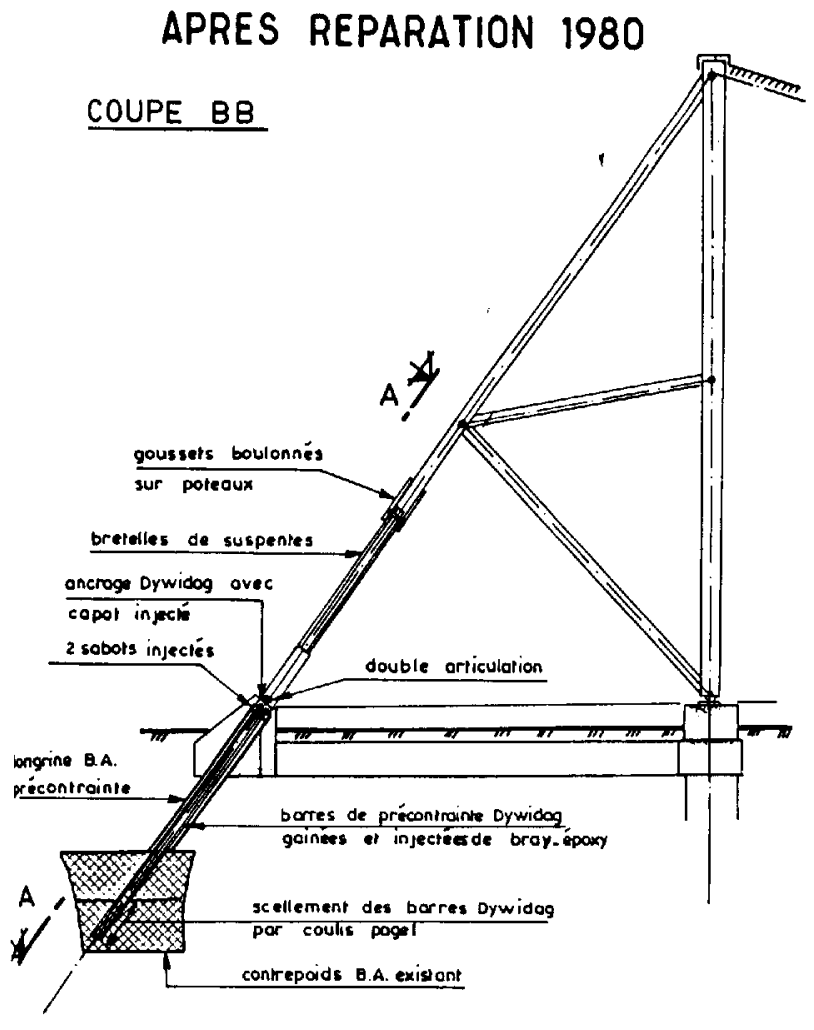

Fig. 9

\section{APRES REPARATION 1980}

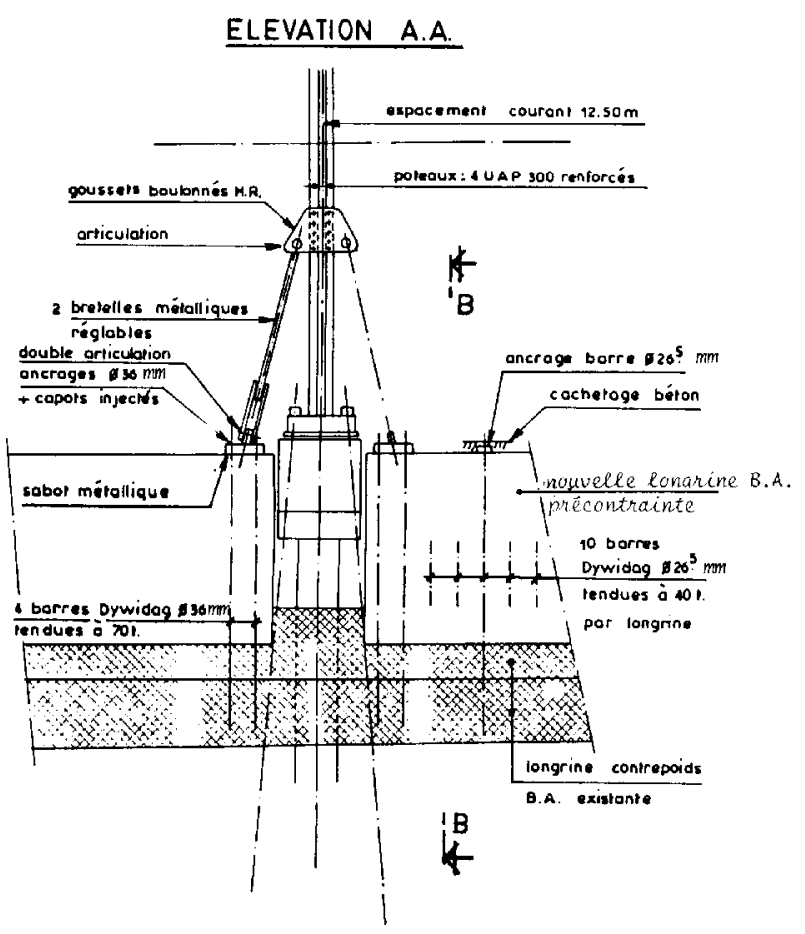

Fig. 10 ments $D, E$ et $D$ ' vers l'intérieur du bâtiment sous l'action des tensions des câbles de toiture. Ces fondations se soulevèrent après la rupture de leurs ancrages.

3. L'origine des désordres est imputable à la rupture par corrosion sous tension des fils $\mathrm{KA}$ de plusieurs tirants d'ancrage précontraints destinés à stabiliser les fondations de la charpente métallique reposant sur deux files de pieux. Ce phénomène de corrosion sous tension, auquel la nature des fils KA est très sensible, a été accéléré par l'absence totale de protection par coulis d'injection en différentes zones des tirants, et plus particulièrement en tête des tirants sous les sabots métalliques à la traversée des massifs de fondations supérieurs, zone où la plupart des ruptures se sont produites. Les défauts de protection contre la corrosion étaient systématiques et se sont retrouvés aussi bien en façade Nord qu'en façade Sud. Il s'agit donc d'un vice généralisé. Seuls, les tirants de renfort du type B.B.R.V., exécutés deux ans après les tirants KA, ont paru correctement protégés contre la corrosion.

4. Le coût des travaux confortatifs a atteint $7616412 \mathrm{~F}$, taxes comprises.

5. De ce sinistre, qui aurait pu devenir une catastrophe avec de nombreux morts, si la présence d'esprit d'un balayeur n'avait pas alerté les techniciens en temps utile, on peut, à notre avis, tirer la philosophie suivante:

- Certaines nouvelles techniques de pointe ne de- vraient être commercialisées qu'après contrôle de l'efficacité de leurs procédés de mise en ceuvre sur une longue période. Dans le cas particulier considéré, il apparaît aujourd'hui que la conception des tirants et les modalités de leur injection ne permettaient pas d'assurer une protection suffisante contre la corrosion fissurante sous tension.

- On sera particulièrement vigilant pendant l'exécution des tirants précontraints actuels. On s'assurera que les coulis d'injection pénètrent correctement sous pression dans toutes les zones à protéger et ce, tout spécialement pour les tirants "permanents " dont l'utilisation se développe de plus en plus depuis quelques années. On installera un système de vérification de tension dans ces tirants permanents. La tension de ces tirants sera contrôlée périodiquement, ce qui permettra de remplacer, si nécessaire, les tirants défaillants.

6. A l'époque de la réalisation des travaux de tirants de cette affaire, il n'existait pratiquement aucun texte de caractère normatif correspondant à cette technique. En 1972, des recommandations ont été publiées sous l'égide du Bureau Securitas (T.A. 72). En 1977, ces recommandations ont faits l'objet d'une nouvelle version amélioré (T.A. 77). Elles sont en cours de réédition sous la forme de T.A. 85. Synthèse de l'expérience de divers partenaires (bureaux de contrôle, entreprises, maîtres d'œuvre publics notamment), ces recommandations ont précisément pour objet d'éviter, dans le futur, de tels sinistres. 